\title{
Microbial contamination of home nebulizers in children with cystic fibrosis and clinical implication on the number of pulmonary exacerbations
}

\author{
Seyed Ahmad Tabatabaii ${ }^{1}$, Ghamartaj Khanbabaee ${ }^{1}$, Saeed Sadr ${ }^{1}$, Nazanin Farahbakhsh ${ }^{2^{*}}$ (D, \\ Maryam Kazemi Aghdam ${ }^{3}$, Saran Lotfollahzadeh ${ }^{4}$, Amirhossein Hosseini ${ }^{5}$, Naghi Dara ${ }^{5}$, \\ Mohammad Nanbakhsh ${ }^{2}$ and Fatemeh Abdollah Gorji ${ }^{6}$
}

\begin{abstract}
Background: Early detection of pulmonary contamination in children with cystic fibrosis (CF) is essential since these children are vulnerable to Pseudomonas aeruginosa (P. aeruginosa) colonization. In Iran, home nebulization of antibiotics is a widespread practice in treatment for patients with CF and, to the best our knowledge, no bacteriological surveys have been conducted till date in this regard.

Method: This observational, cross sectional study was conducted on 61 children with CF at Mofid Children's Hospital, Tehran, from September 2017 to march 2018. The swab sampling was performed from 61 home nebulizers used by children diagnosed with CF. Contemporaneous sputum sample or deep nasopharyngeal swab was taken from each patient for bacterial and fungal testing. Medical records of the patients were reviewed and the number of exacerbations were recorded over the last 12 months prior to the study enrollment.
\end{abstract}

Results: The results of study showed that, 43 (70.5\%) nebulizers were contaminated; 31 (50.8\%) mouthpieces, 21 (34.4\%) reservoirs, and 11 (18\%) connecting tubes. The most common organism to be isolated was $P$. aeruginosa and was recovered from 19 (31\%) nebulizers, 16 of them belonged to patients chronically colonized with $P$. aeruginosa. The remaining three had at least one positive sputum culture for $P$. aeruginosa in the past 1 year before the study. There was a significant increase in the number of CF exacerbations with an average number of exacerbation being $1.5 \pm 1$ (SD) over last 12 months in children who had pathogenic organisms recovered from their home nebulizers compared with $0.4 \pm 0.7(\mathrm{SD})$ exacerbations per year in whom non-pathogenic organisms were isolated from their nebulizers $(P<0.001)$.

Conclusion: The majority of domiciliary nebulizers used by children with CF were contaminated with microorganisms indicating that the nebulizers may serve as potential reservoirs of pathogens for the patients' lung. Perpetuating colonization is a possible concern in the ones recently colonized with $P$. aeruginosa and, therefore, decontamination of nebulizer requires more attention to prevent ongoing infection. The negative impact of contamination of nebulizer on CF exacerbation requires serious attention and further investigations.

Keywords: Contamination, Cystic fibrosis, Microorganism, Nebulizer, Exacerbation

\footnotetext{
* Correspondence: nazanin26farahbakhsh@yahoo.com

${ }^{2}$ Department of Pediatric Pulmonology, Mofid Children's Hospital, Shahid

Beheshti University of Medical Sciences, Tehran, Iran

Full list of author information is available at the end of the article
}

(c) The Author(s). 2020 Open Access This article is distributed under the terms of the Creative Commons Attribution 4.0 International License (http://creativecommons.org/licenses/by/4.0/), which permits unrestricted use, distribution, and reproduction in any medium, provided you give appropriate credit to the original author(s) and the source, provide a link to the Creative Commons license, and indicate if changes were made. The Creative Commons Public Domain Dedication waiver (http://creativecommons.org/publicdomain/zero/1.0/) applies to the data made available in this article, unless otherwise stated. 


\section{Background}

Cystic fibrosis (CF) is a life-threatening genetic disorder with an autosomal recessive pattern of inheritance [1]. This multisystem disease is caused by mutations in the CF transmembrane conductance regulator (CFTR) gene located on chromosome 7 [2]. The common manifestations of the disease are variable and include recurrent and persistent pulmonary infection, pancreatic insufficiency, and abnormal sweat chloride levels [3]. Although the disease have multisystem involvement, pulmonary manifestations account for increased morbidity and mortality in these patients [4].

Pulmonary infection by various microorganisms is a common presentation and these children sometimes have persistent bacterial infection within their lungs [3]. The common organisms, regardless of age, include Pseudomonas aeruginosa ( $P$. aeruginosa), Burkholderia cepacia (B. cepacia), Staphylococcus aureus (S. aureus), and methicillin-resistant Staphylococcus aureus (MRSA). Colonization with these microorganisms results in a rapid deterioration of lung functions, thus increasing the risk of mortality [5-7]. Nebulized drugs (mucolytics and antibiotics) are essential components in the management of pulmonary infections [8]. Nebulization of antibiotics delivers high drug concentrations directly to the lungs, while decreasing the systemic adverse effects of these drugs over kidneys, liver, and auditory system [3]. The introduction of home nebulizers allows the use of this therapy at home and avoids the unnecessary hospital visits and admissions.

Early detection and eradication of the sources causing pulmonary contamination is the main concern of all pulmonologists, as the patients with CF are vulnerable to colonization with pathogenic organisms such as $P$. aeruginosa $[9,10]$. Several cohort studies suggested that the contamination of equipment, such as home nebulizers, could serve as a reservoir for microorganisms, particularly Pseudomonas species leading to colonization of lungs and pulmonary infection [11-13]. Nebulizers generate very small particles, which can easily reach the terminal bronchioles and alveoli; therefore, nebulizer organism reaches the lungs easily, which may lead to pulmonary colonization. This highlight the importance of cleaning of home nebulizers [14]. Nebulizer contamination and the clinical implications are not well studied in the young age children with CF, as dependency on the caregivers and parents, and the chronicity of the disease may affect good hygiene of nebulizers.

In Iran, home administration of nebulized antibiotics is widespread as a treatment for children with CF. To the best of our knowledge, no surveys of bacteriological contamination of these nebulizers have been conducted in Iran. There are only a few international articles, that too with small sample sizes $[11,15,16]$. We studied the microbial contamination of home nebulizers used by children with $\mathrm{CF}$ and the findings were correlated with the children's sputum culture results. We also sought to study the clinical implication of microbial contamination of home nebulizers, which has not been studied previously.

\section{Methods}

\section{Patient selection}

This observational cross sectional study was conducted at Mofid Children's Hospital, Tehran, from September 2017 to March 2018. The study enrolled 61 children diagnosed with CF who were routinely followed up in the hospital. Sample size was calculated according to the study by Blau et al. [13]. Written informed consent was taken from patients/parents before enrollment in the study. Inclusion criteria were a definitive diagnosis of CF based on positive results of two sweat chloride tests, and administration of home nebulizers at least once a day. The exclusion criteria were patients receiving intravenous (IV) antibiotics at the time of recruitment, using an inhaled antibiotic on the day of sample collection, patients not using their home nebulizers daily or whose parents refused to consent for study participation.

The patients received 5\% hypertonic saline (as mucolytic), bronchodilators, and if necessary, an antibiotic (orally or inhaled through nebulizer). All patients who were regularly followed up were asked to bring their home nebulizers with a facial mask/oral piece, and connectors on their scheduled outpatient visit, they were instructed to clean the nebulizer after use the night prior to the visit.

Demographic data of patients were also collected at the same visit. Medical records of patients were reviewed and recorded for the current colonization, past or current antibiotic use, number of exacerbations, use of oral systemic antibiotics and the requirement for hospitalization and IV antibiotics during the past 12 months. A contemporaneous sputum sample or deep nasopharyngeal swab, in subjects unable to produce sputum, was also taken.

In the current study, there were no missing data due to the referral policies for all outpatient and inpatients. Authors had access to the outpatient and inpatient records of the enrolled cases.

\section{Hygiene and maintenance of nebulizers}

Each patient in the study population had their own personal domestic nebulizer. Families were interviewed through a checklist about hygiene and maintenance of nebulizers including frequency and method of cleaning, disinfecting, and drying. After filling up the checklist by a pediatric pulmonologist, the proper method of cleaning, disinfecting and maintenance of the nebulizer was 
also explained to them. In the study center, the CF Foundation's recommended nebulizer care is followed [17] .

\section{Cultures}

A sterile cotton swab moistened with sterile saline was rotated multiple times inside the mouthpiece or mask, reservoir, and connectors separately in a clockwise direction. Then, the swabs were immediately smeared on the surface of blood agar, chocolate agar, and MacConkey agar plates. The plates were incubated at $37^{\circ} \mathrm{C}$ for 3 days. They were checked for colony formation every 24 $\mathrm{h}$ till $72 \mathrm{~h}$. The average colony-forming units (CFUs) for each cultured surface were reported by the same pathologist. Surfaces with less than 10 CFUs were considered clean, according to the study by Blau et al. [13]. In vitro susceptibility testing was performed on all Pseudomonas spp. isolated either from sputum or devices by disk diffusion method and interpreted according to the criteria published by the Clinical Laboratory Standards Institute (CLSI) 2017 [18]. P. aeruginosa and S. aureus were considered as the pathogenic floras.

\section{Statistical analysis}

Statistical analysis was performed using SPSS version 19 for windows (Chicago, IL). The categorical outcome variables were analyzed by the Chi-square test with Fisher's exact test. Continuous variables were analyzed by Student's t-test or Mann-Whitney U test after assessment of normality of data using the Kolmogorov-Smirnov test as applicable. $P$ values of less than 0.05 was taken as significant.

\section{Results}

During the study period 61 children diagnosed with CF were evaluated. All patients had only one reusable home nebulizer. The baseline characteristics and data regarding patients and home nebulizers are summarized in Table 1.

The respiratory colonization profile of the children examined through culturing contemporaneous sputum samples or deep pharyngeal swabs showed a predominance of chronic colonization with $P$. aeruginosa (Fig. 1). Out of the 61 evaluated nebulizers, 39 (63.9\%) were jet nebulizers and 43 (70.5\%) nebulizers were contaminated (any part). The contaminated parts of the nebulizers included reservoirs (65.6\%), mouthpieces (49.2\%), and connectors (18\%). In six nebulizers, all the three evaluated parts were contaminated; in eight nebulizers, only two parts were contaminated, and in 29 nebulizers, the microorganism was recovered only from one part. Multiple species contamination was observed in six devices. A wide variety of microorganisms were isolated from nebulizer cultures, of which $P$. aeruginosa was predominant $(n=19)$ (Fig. 2).
Table 1 Baseline Characteristics of Patients and Home Nebulizers

\begin{tabular}{ll}
\hline Data regarding patients/ home nebulizers & $\begin{array}{l}\text { Mean } \pm \text { SD or } \mathrm{n}(\%) \\
\text { Total }=61\end{array}$ \\
\hline Age (years) & $7.6 \pm 4.2$ \\
Weight (Kg) & $23.6 \pm 13.3$ \\
Height / length (cm) & $121.3 \pm 25.3$ \\
Male gender (\%) & $35(57.4 \%)$ \\
Type of home nebulizers (\%) & \\
Jet & $39(63.9 \%)$ \\
Ultrasonic & $16(26.2 \%)$ \\
Mesh & $6(9.8 \%)$ \\
Duration of use (years) & $3.6 \pm 2.9$ \\
Replace nebulizer parts & \\
None & $48(78.5 \%)$ \\
Mask/mouthpiece & $6(10 \%)$ \\
Reservoir & $4(6.5 \%)$ \\
Connecting tube & $3(5 \%)$ \\
Number of daily inhalation & \\
All devices & $2.6 \pm 0.7$ \\
Contaminated devices & $2.7 \pm 0.7$ \\
Clean devices & $2.3 \pm 0.8$ \\
Inhaled antibiotics (\%) & \\
None & $18(13.1 \%)$ \\
Gentamicin & $23(37.7 \%)$ \\
\hline Vancomacin & \\
\hline
\end{tabular}

Comparison of nebulizers' contamination with sputum culture (Table 2) showed that 19 nebulizers were contaminated with Pseudomonas spp., sixteen (84.2\%) of which belonged to the children whose contemporaneous sputum cultures were positive for $P$. aeruginosa; this relationship was statistically significant $(P<0.001)$. Five nebulizers showed growth of $S$. aureus, two (40\%) of these belonged to patients whose contemporaneous sputum cultures were positive for $S$. aureus $(P<0.001)$. Eleven nebulizers showed growth of coagulase-negative staphylococci (CoNS), of which one (9.5\%) nebulizer belonged to a child whose contemporaneous sputum culture showed growth of CoNS $(P=0.455)$. Thirteen nebulizers were contaminated with micrococci, of which two (15.4\%) children had growth of micrococci from their contemporaneous sputum cultures $(P=0.043)$. Klebsiella spp. and Escherichia coli were not isolated from any of the nebulizers. Two nebulizers were contaminated with Enterobacter spp., of which one (50\%) nebulizer belonged to a child that his contemporaneous sputum culture positive for Enterobacter spp.; however, 


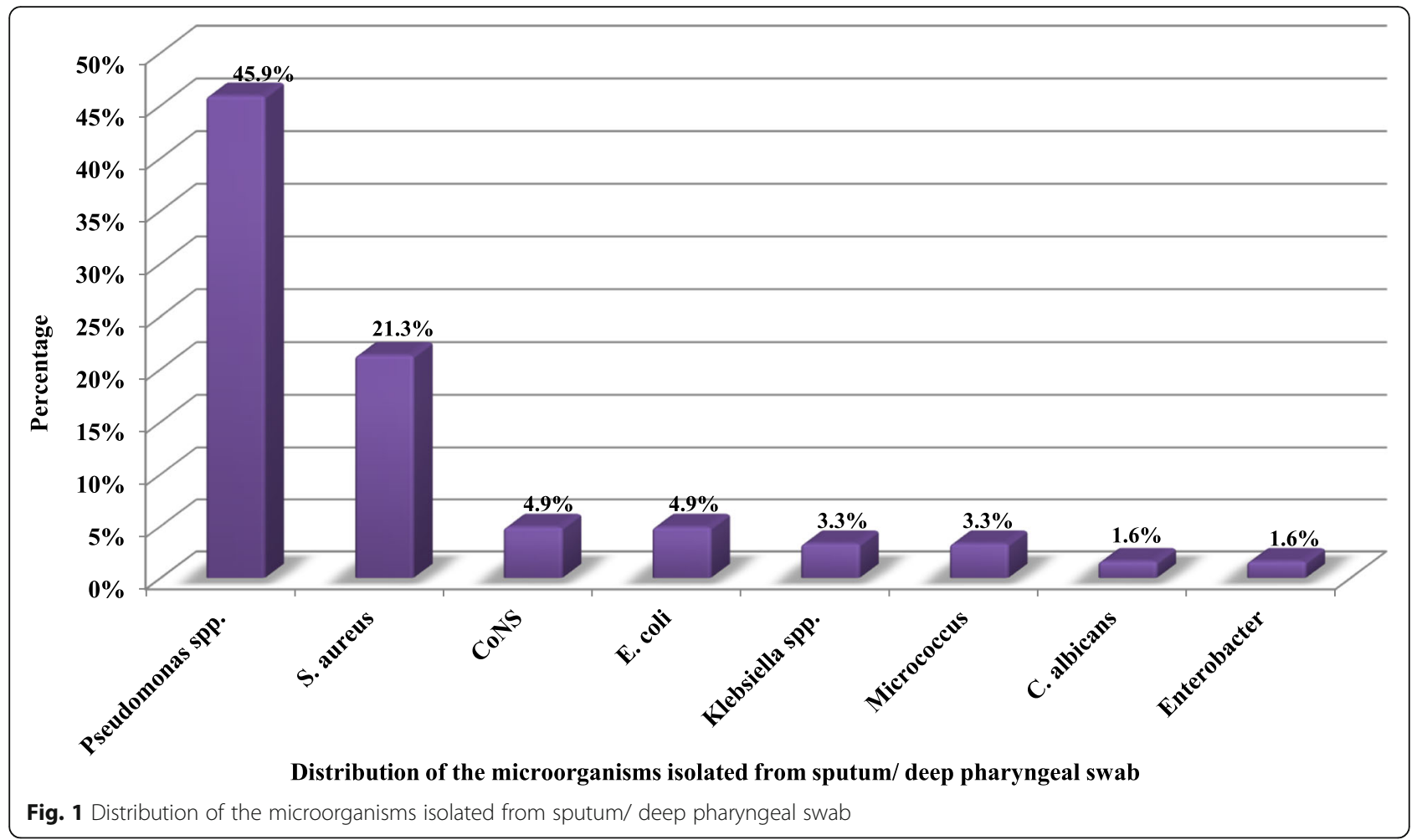

the relationship was significant $(P=0.033)$. Nebulizer and sputum culture results didn't have a significant relationship for growth of Candida albicans $(P=0.066)$. The overall correlation of sputum and nebulizer cultures was 93\% $(P<0.001)$.
The number of daily inhalation was reported in the present study; the mean number of inhalation per day for contaminated and clean devices were $2.39 \pm 0.7$ (SD) and $2.67 \pm 0.8(\mathrm{SD})$ inhalation per day respectively, which was not statistically significant $(P=0.095)$. Among the

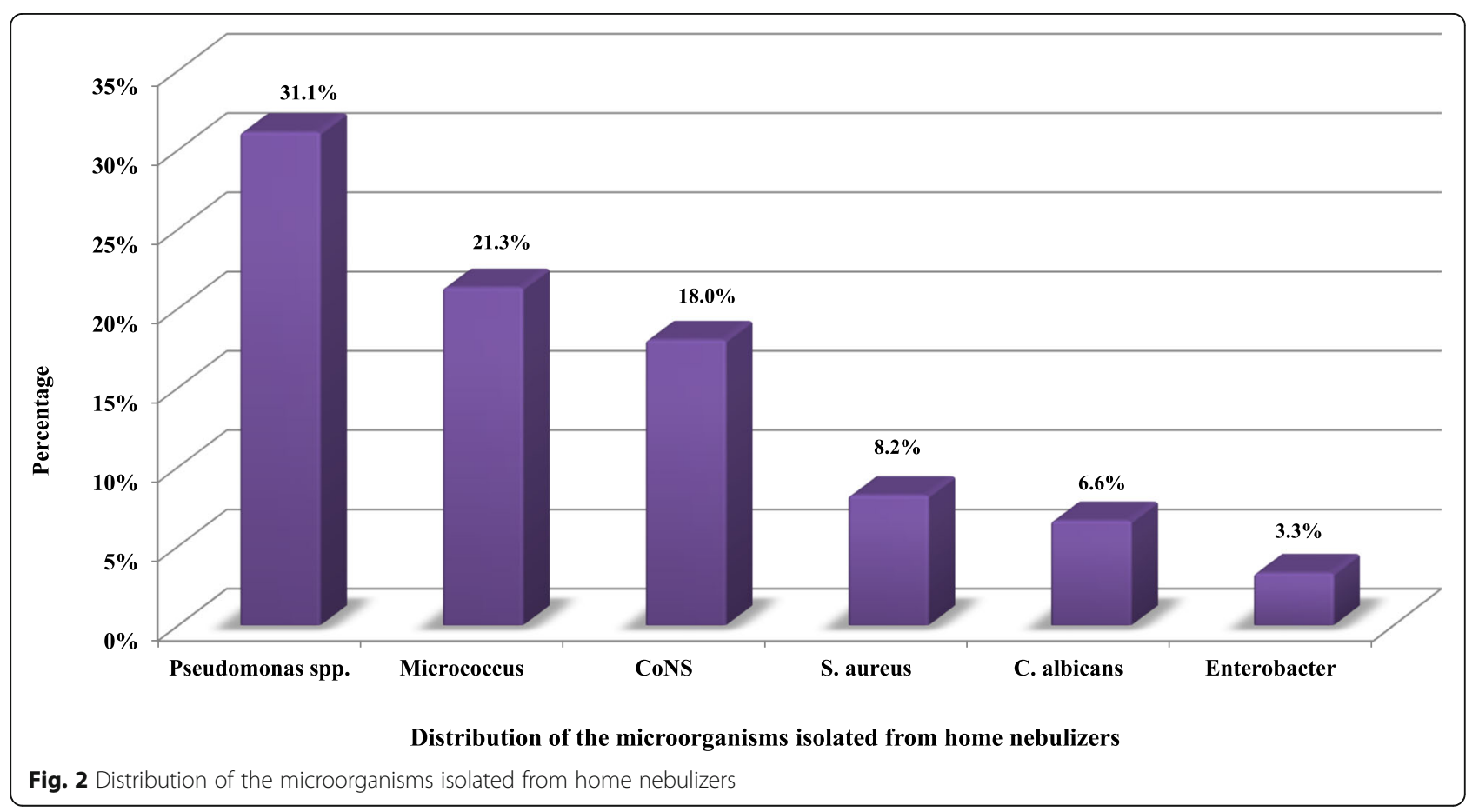


Table 2 Table Showing Relationship Between Nebulizers Contamination and Sputum Culture Results

\begin{tabular}{llllll}
\hline Microorganism in Sputum & & \multicolumn{2}{l}{ Nebulizer } & P-value \\
\cline { 2 - 4 } & & Positive & Negative & \\
\hline Pseudomonas spp. & Positive & 16 & 12 & $<0.001$ \\
Staphylococcus aureus & Negative & 3 & 30 & \\
& Positive & 2 & 11 & $<0.001$ \\
Coagulase negative staphylococci & Negative & 3 & 45 & \\
Micrococcus spp. & Posive & 1 & 2 & 0.455 \\
& Negative & 10 & 48 & \\
Enterobacter spp. & Positive & 2 & 0 & 0.043 \\
& Negative & 11 & 48 & \\
& Positive & 1 & 0 & 0.033 \\
& Negative & 1 & 59 & \\
\hline
\end{tabular}

38 patients who received inhaled daily antibiotics, 32 (84.2\%) of them had culture growth from their devices. Of the remaining 23 patients who were not on any inhaled antibiotic therapy, only 11 (48\%) had culture growth from their devices. The estimated risk for positive device culture was significantly higher in those receiving inhaled daily antibiotics when compared to those who were not receiving $(P=0.004)$.

Among 61 home nebulizers, 24 were contaminated with pathogenic flora ( $P$. aeruginosa and $S$. aureus), 19 cultured non- pathogenic flora and 18 cultured no organism. Patients' medical records were investigated in order to obtain the number of CF exacerbations which ended in hospital admission and IV antibiotics during the past 12 months. The mean number of exacerbations was $1.5 \pm 1(\mathrm{SD})$ per year in patients whose nebulizers were contaminated with the pathogenic flora (named as pathogenic flora group) and $0.4 \pm 0.7(\mathrm{SD})$ exacerbations per year in the non-pathogenic flora group $(P<0.001)$ (Table 3).

The data regarding the hygiene and maintenance of nebulizers have been summarized in Table 4. All the patients/families did the cleaning step properly. Unfortunately, $54.1 \%$ of the patients/families were not aware of proper disinfection procedure and only $45.9 \%$ of nebulizers were disinfected prior to drying. The majority of the nebulizers $(86.9 \%)$ were air dried at final step. Only $50.8 \%$ of the subjects did the process of washing nebulizer after each use, which is considered appropriate frequency according to CF foundation. Considering the
Table 4 Table Showing the Data Regarding the Nebulizers' Hygiene and Maintenance

\begin{tabular}{ll}
\hline $\begin{array}{l}\text { Data regarding } \\
\text { nebulizers' Hygiene }\end{array}$ & $\mathrm{n}(\%)$ \\
\hline Cleaning step & total =61 \\
None & - \\
Tap Water & $47(77 \%)$ \\
Hot water & $6(9.8 \%)$ \\
Detergent & $8(13.1 \%)$ \\
Disinfecting step & \\
None & \\
Alcohol & $33(54.1 \%)$ \\
Boiling water & $4(6.5 \%)$ \\
Hot water & $16(26.2 \%)$ \\
Drying step & $8(13.2 \%)$ \\
None & \\
Air dry & - \\
Towel & $53(86.9 \%)$ \\
Tissue paper & $3(5 \%)$ \\
Frequency step & $5(8.1 \%)$ \\
After each inhalation & \\
Once a day & \\
Once a week & \\
Arregularly & $31(50.8 \%)$ \\
Correct & $19(31.2 \%)$ \\
\hline & $5(8.2 \%)$ \\
\hline & $6(9.8 \%)$ \\
\hline & $45(73.8 \%)$ \\
\hline
\end{tabular}

whole process of cleaning and maintenance of nebulizers (frequency, cleaning, disinfection, and drying), only 16 (26.2\%) patients/families did the whole process properly. Among the subjects that improperly observed the hygiene of nebulizers, $51.1 \%$ were male and $48.9 \%$ female $(P=0.142)$. The mean age of the subjects was higher in households where nebulizer hygiene was inadequate than in households where nebulizers were maintained following proper procedures $[8.4 \pm 4.1(\mathrm{SD})$ year vs $5.3 \pm$ 3.5(SD) year; $P<0.001]$.

\section{Discussion}

CF-related lung disease includes chronic colonization or infection of the respiratory tract $[1,3]$. Contamination of home nebulizers has been reported in patients with $\mathrm{CF}$

Table 3 Comparison of the Pathogen and Non-Pathogen Microorganisms Isolated from Nebulizers Based on the Number of Exacerbations per Year

\begin{tabular}{lccc}
\hline Variable & $\begin{array}{c}\text { Pathogenic Flora } \\
\text { Mean } \pm \text { SD }\end{array}$ & $\begin{array}{c}\text { Non-Pathogen Flora } \\
\text { Mean } \pm \text { SD }\end{array}$ & $P$ value \\
\hline Number of Exacerbations per year & $1.5 \pm 1.0$ & $0.4 \pm 0.7$ & $<0.001$ \\
\hline
\end{tabular}


$[11,19]$ and we are reporting this type of study from Iran. In the past, patients with CF required hospital stay; however, nowadays they receive inhaled medications via home nebulizers; nevertheless, the nebulizer hygiene is problematic [16].

The present study demonstrated that home nebulizers used by children with CF were predominantly contaminated (70.49\%). Nebulizer contamination observed in the current study was close to the findings reported in a study by Hutchinson et al. (68.8\%) [11], but significantly higher than the results of Della Zuana et al. [16]. This result difference could be because of different country study population and level of knowledge regarding hygiene and maintenance of the nebulizer. In the study conducted by Vassal et al. [20], home nebulizers of 44 patients with CF chronically colonized with $P$. aeruginosa were evaluated; the results indicated that 30 (68\%) nebulizers were contaminated with bacteria immediately after drug nebulization. However, the results of study by Brzezinski et al., [15] on 28 home nebulizers revealed that only $21 \%$ of nebulizers were contaminated with CFrelated bacteria, and no specific microorganism was predominant. This lower rate of nebulizer contamination in study of Brzezinski et al. could be because the sputum smear collection was performed at home, and the samples were kept at room temperature prior to analysis, which would have changed the results of cultures [15]. The strength point of the current study was that the samples were smeared on the surface of appropriate media immediately after collection.

The number of daily inhalation was reported in the present study, as the frequency of nebulizer administration was likely to influence the risk of contamination. However, the mean number of inhalation per day for contaminated and clean devices were not statistically significant $(P=0.095)$. The majority of the home nebulizers analyzed in the current study were not replaced for a long time, so the mean duration of use of a single device was 3.6 years which was higher than the result reported by Brzezinski et al. [15]. The prolonged duration of administrating home nebulizers may cause some fissures in the device surface and increase the chance of contamination, therefore these nebulizers should be replaced after fixed duration of time as recommended by manufacturers.

The main microorganism in the current study was $P$. aeruginosa, isolated from both nebulizers and patients' respiratory secretions. Comparing with the previous bacteriological survey published from our same center, the number of patients with CF colonized with Pseudomonas spp. increased during the last 6 years from 38.8 to $45.9 \%$ [21]. This highlights the importance of screening and eradicating $P$. aeruginosa strains. The current study results demonstrated a significant correlation between microorganisms isolated from sputum cultures and nebulizer samples $(n=51 ; 83.6 \%)$ which is in contrast to the findings of the study published by Rosenfeld et al., [22] who reported that the domiciliary nebulizers used by children with CF were respectively contaminated with S. aureus (55\%), P. aeruginosa (35\%), and Klebsiella spp. (19\%) in their study population and the correlation between the bacteria isolated from devices and sputum samples was poor. Brzezinski et al., similarly demonstrated no association between the result of sputum and nebulizer cultures [15]. The current study, similar to other studies, could not determine if patients or nebulizers were the primary sources of infection; however, it was noted that higher contamination rate of home nebulizers, especially with Pseudomonas spp., increased the risk of reintroduction or perpetuation of infection [19, 20]. In the current study, 19 evaluated nebulizers were contaminated with Pseudomonas spp. (at any parts); 16 of them belonged to patients chronically infected with $P$. aeruginosa, and the remaining three showed no growth of $P$. aeruginosa on sputum cultures during the study, but had at least one positive sputum culture for $P$. aeruginosa during the past 1 year prior to the study. It was similar to the results of study published by Blau et al. in which all the nebulizers contaminated with Pseudomonas spp. belonged to patients chronically colonized with $P$. aeruginosa [13]. The genetic identification of the bacterial strains was beyond the aim of study and we plan to conduct such study in near future. Due to study limitations, the antibiogram was only performed for Pseudomonas spp. isolated from the sputum samples and nebulizers; in 16 cases of which the Pseudomonas spp. was recovered from sputum sample and nebulizer concomitantly, 13 of them showed similarity in antibiogram with each other. In the remaining three whose antibiogram results were not similar, CoNS was also isolated simultaneously from the devices, therefore increasing the possibility of surface contamination.

The patients were divided into three groups based on the results of nebulizer cultures (pathogen, nonpathogen, no microorganism), in order to evaluate the impact of device contamination on clinical outcomes. There was a significant increase in the frequency of exacerbations that ended in hospital admission over 12 months in children from whom pathogenic bacteria were recovered from their home nebulizers $(P<0.001)$. This novel finding has been reported for the first time in a population of children with CF. It should be considered that pulmonary exacerbations have negative consequences on children's lung function $[23,24]$ and imposes a significant burden on family and the health care system.

According to the current study results, with the increase in age of children maintenance and hygiene of 
nebulizers decreases. This can be due to the chronicity of CF, decrease in parents' supervision over time and poor treatment compliance. This highlights the importance of continuous education for parents'/caregivers in every outpatient visit.

\section{Conclusion}

Majority of domiciliary nebulizers in children with CF are contaminated with microorganisms. Further educational programs regarding the nebulizer hygiene in $\mathrm{CF}$ children is required. The increased incidence of exacerbations in children using pathogen-contaminated nebulizers may reflect non-adherence to the hygiene of nebulizers. However, autoinfection should be considered in the patients recently colonized with $P$. aeruginosa. The negative impact of nebulizer contamination on exacerbations requires more attention and further investigations.

\section{Limitations and strength of the study}

The current study had some limitations such as small sample size, and no facility for genetic identification of bacterial strains and antibiogram testing for all positive cultures. The strength of the study includes immediately putting the sputum obtained from the patients for culture thus yielding high rate of organism growth, uniform follow of protocol and no lost to follow up of the enrolled subjects.

Obviously, the level of socioeconomic status can affect hygiene and the maintenance and hygiene of home nebulizers is not exempt from this issue. Also, the burden of any disease can affect the quality of care, treatment adherence and hygiene.

\section{Abbreviations \\ C. albicans: Candida albicans; CF: Cystic fibrosis; CFU: Colony forming unit; CoNS: Coagulase negative staphylococci; E. coli: Escherichia coli; S. aureus: Staphylococcus aureus}

\section{Acknowledgments}

The authors feel obliged to thank the Pediatric Pathology Research Center, Research Institute for Children Health at Shahid Beheshti University of Medical Sciences for their support of this project. We also thank the patients for cooperation who participated in this study.

\section{Authors' contributions}

SAT and NF participate in research design, the acquisition of data, the writing of the manuscript, and the performance of the research. GK, SS, MKA, $S L, F A G$ and $A H H$, contributed to the acquisition of data, interpretations of data, NF and MN participate in preparation of the manuscript and final revision. All authors read and approved the final revision of the manuscript.

\section{Funding}

No funding was obtained for this study or writing the manuscript.

\section{Availability of data and materials}

The datasets used and/or analyzed during the current study are available from the corresponding author on reasonable request.

\section{Ethics approval and consent to participate}

Informed written consent was taken from parents and/or patients and the study was approved by the ethical committee of Shahid Beheshti University of Medical Sciences with the ethics code of IR.SBMU.RETECH.REC.1396.937.

Consent for publication

Not applicable.

\section{Competing interests}

The authors declare that they have no competing interests.

\section{Author details}

'Department of Pediatric Pulmonology, Mofid Children's Hospital, Shahid Beheshti University of Medical Sciences, Tehran, Iran. ${ }^{2}$ Department of Pediatric Pulmonology, Mofid Children's Hospital, Shahid Beheshti University of Medical Sciences, Tehran, Iran. ${ }^{3}$ Pediatric Pathology Research Center, Research Institute for Children's Health, Shahid Beheshti University of Medical Sciences, Tehran, Iran. ${ }^{4}$ Pediatric surgery Research Center, Research Institute for Children's Health, Shahid Shahid Beheshti University of Medical Sciences, Tehran, Iran. ${ }^{5}$ Pediatric Gastroenterology, Hepatology and Nutrition Research Center, Research Institute for Children's Health, Shahid Beheshti University of Medical Sciences, Tehran, Iran. ${ }^{6}$ Medical Research Development Center, Mofid Children's Hospital, Shahid Beheshti University of Medical Sciences, Tehran, Iran.

Received: 20 January 2019 Accepted: 23 January 2020

Published online: 06 February 2020

\section{References}

1. Ratjen F, Doring G. Cystic fibrosis. Lancet. 2003;361(9358):681-9.

2. Farjadian S, Moghtaderi M, Kashef S, Alyasin S, Najib K, Saki F. Clinical and genetic features in patients with cystic fibrosis in southwestern Iran. Iran J Pediatr. 2013;23(2):212-5

3. Gibson RL, Burns JL, Ramsey BW. Pathophysiology and management of pulmonary infections in cystic fibrosis. Am J Respir Crit Care Med. 2003; 168(8):918-51.

4. VanDevanter DR, Kahle JS, O'Sullivan AK, Sikirica S, Hodgkins PS. Cystic fibrosis in young children: a review of disease manifestation, progression, and response to early treatment. J Cyst Fibros. 2016;15(2):147-57.

5. Fajac I, Wainwright CE. New treatments targeting the basic defects in cystic fibrosis. Presse Med. 2017:46(6 Pt 2):e165-e75.

6. Zemanick ET, Harris JK, Conway S, Konstan MW, Marshall B, Quittner AL, et al. Measuring and improving respiratory outcomes in cystic fibrosis lung disease: opportunities and challenges to therapy. J Cyst Fibros. 2010;9(1):1-16.

7. Boucher RC. Airway surface dehydration in cystic fibrosis: pathogenesis and therapy. Annu Rev Med. 2007:58:157-70.

8. Arias Llorente RP, Bousono Garcia C, Diaz Martin JJ. Treatment compliance in children and adults with cystic fibrosis. J Cyst Fibros. 2008;7(5):359-67.

9. Gaspar MC, Couet W, Olivier JC, Pais AA, Sousa JJ. Pseudomonas aeruginosa infection in cystic fibrosis lung disease and new perspectives of treatment: a review. Eur J Clin Microbiol Infect Dis. 2013;32(10):1231-52.

10. Bhagirath AY, Li Y, Somayajula D, Dadashi M, Badr S, Duan K. Cystic fibrosis lung environment and Pseudomonas aeruginosa infection. BMC Pulm Med. 2016;16(1):174.

11. Hutchinson GR, Parker S, Pryor JA, Duncan-Skingle F, Hoffman PN, Hodson $\mathrm{ME}$, et al. Home-use nebulizers: a potential primary source of Burkholderia cepacia and other colistin-resistant, gram-negative bacteria in patients with cystic fibrosis. J Clin Microbiol. 1996;34(3):584-7.

12. Cohen HA, Kahan E, Cohen Z, Sarrell M, Beni S, Grosman Z, et al. Microbial colonization of nebulizers used by asthmatic children. Pediatr Int. 2006;48(5): 454-8.

13. Blau H, Mussaffi H, Mei Zahav M, Prais D, Livne M, Czitron BM, et al. Microbial contamination of nebulizers in the home treatment of cystic fibrosis. Child Care Health Dev. 2007;33(4):491-5.

14. Carvalho TC, Peters JI, Williams RO 3rd. Influence of particle size on regional lung deposition--what evidence is there? Int J Pharm. 2011;406(1-2):1-10.

15. Brzezinski LX, Riedi CA, Kussek P, Souza HH, Rosario N. Nebulizers in cystic fibrosis: a source of bacterial contamination in cystic fibrosis patients? J Bras Pneumol. 2011;37(3):341-7. 
16. Della Zuana A, Garcia Dde O, Juliani RC, Silva Filho LV. Effect that an educational program for cystic fibrosis patients and caregivers has on the contamination of home nebulizers. J Bras Pneumol. 2014;40(2):119-27.

17. Saiman $L$, Siegel J. Infection control recommendations for patients with cystic fibrosis: microbiology, important pathogens, and infection control practices to prevent patient-to-patient transmission. Infect Control Hosp Epidemiol. 2003;24(5 Suppl):S6-52.

18. Clinical and Laboratory Standards Institute. Performance Standards for Antimicrobial Susceptibility Testing; Twenty-Seventh Informational Supplement. CLSI Document M100-S27. Wayne, PA: Clinical and Laboratory Standards Institute; 2017.

19. Pitchford KC, Corey M, Highsmith AK, Perlman R, Bannatyne R, Gold R, et al. Pseudomonas species contamination of cystic fibrosis patients' home inhalation equipment. J Pediatr. 1987;111(2):212-6.

20. Vassal S, Taamma R, Marty N, Sardet A. d'athis P, Bremont F, et al. microbiologic contamination study of nebulizers after aerosol therapy in patients with cystic fibrosis. Am J Infect Control. 2000;28(5):347-51.

21. Khanbabaee G, Akbarizadeh M, Sayyari A, Ashayeri-Panah M, Abdollahgorji F, Sheibani K, et al. A survey on pulmonary pathogens and their antibiotic susceptibility among cystic fibrosis patients. Braz J Infect Dis. 2012;16(2):122-8.

22. Rosenfeld M, Joy P, Nguyen CD, Krzewinski J, Burns JL. Cleaning home nebulizers used by patients with cystic fibrosis: is rinsing with tap water enough? J Hosp Infect. 2001;49(3):229-30

23. Waters V, Ratjen F. Pulmonary exacerbations in children with cystic fibrosis. Ann Am Thorac Soc. 2015;12(Suppl 2):S200-6.

24. Hoppe JE, Wagner BD, Sagel SD, Accurso FJ, Zemanick ET. Pulmonary exacerbations and clinical outcomes in a longitudinal cohort of infants and preschool children with cystic fibrosis. BMC Pulm Med. 2017;17(1):188.

\section{Publisher's Note}

Springer Nature remains neutral with regard to jurisdictional claims in published maps and institutional affiliations.

Ready to submit your research? Choose BMC and benefit from:

- fast, convenient online submission

- thorough peer review by experienced researchers in your field

- rapid publication on acceptance

- support for research data, including large and complex data types

- gold Open Access which fosters wider collaboration and increased citations

- maximum visibility for your research: over $100 \mathrm{M}$ website views per year

At $\mathrm{BMC}$, research is always in progress.

Learn more biomedcentral.com/submissions 